\title{
Research Letter \\ Study of Magnesium Diboride Clusters Using Hybrid Density Functional Theory
}

\author{
D. Rodríguez, D. Soto, E. Ramírez, A. Cruz, A. Santana, and G. E. López \\ Theoretical Chemistry and Computational Group, Department of Chemistry, University of Puerto Rico at Mayagüez, \\ Mayagüez, PR 00681, USA \\ Correspondence should be addressed to G. E. López, glopez@uprm.edu
}

Received 25 September 2007; Accepted 31 October 2007

Recommended by Angel Rubio Secades

Using hybrid density functional theory and a relatively large basis set, the lowest energy equilibrium structure, vibrational spectrum, and natural orbital analysis were obtained for magnesium diboride clusters $\left[\left(\mathrm{MgB}_{2}\right)_{x}\right.$ for $x=1,2$, and 3]. For comparison, boron clusters $\left[\mathrm{B}_{x}\right.$ for $x=2,4$, and 6] were also considered. The $\mathrm{MgB}_{2}$ and $\left(\mathrm{MgB}_{2}\right)_{2}$ showed equilibrium structures with the boron atoms in arrangements similar to what was obtained for pure boron atoms, whereas, for $\left(\mathrm{MgB}_{2}\right)_{3}$ a different arrangement of boron was obtained. From the population analysis, large electron density in the boron atoms forming the clusters was observed.

Copyright (C) 2008 D. Rodríguez et al. This is an open access article distributed under the Creative Commons Attribution License, which permits unrestricted use, distribution, and reproduction in any medium, provided the original work is properly cited.

\section{INTRODUCTION}

Materials composed of diborides have attracted the attention of researchers in recent years because of itssuperconductivity properties $[1,2]$. In particular, it has been found that $\mathrm{MgB}_{2}$ behaves as a superconductor at relatively high critical temperature, $T_{\mathrm{c}}=40 \mathrm{~K}$. Although $T_{\mathrm{c}}$ for this material is not as high as other oxides with superconducting properties, its preparation is less expensive and it has a lower anisotropy, making smoother grain boundaries and hence increasing current flow. The origin of the superconducting properties of $\mathrm{MgB}_{2}$ is still a topic of debate [3]. However, the structure of $\mathrm{MgB}_{2}$ might have a significant importance in this property. This material shows a simple hexagonal $\mathrm{AlB}_{2}$-type structure typical of borides. It is formed of layers of boron similar to the basal plane of graphite, separated by closed-packed layers of magnesium. The magnesium atoms are located at the center of the hexagons formed by the boron atom and donate the electron density to the boron layers. It has been postulated [4] that this electron donation might be related to the superconducting nature of this material. Also, electronic structure calculations of bulk $\mathrm{MgB}_{2}$ have shown [5] a metallic character of the material which causes a strong electronphonon interaction and hence superconducting properties. Experimental evidence has suggested [4] that an increment in the $T_{\mathrm{c}}$ temperature can be obtained if nanosized particles of $\mathrm{MgB}_{2}$ are embedded in inert materials. This increment in $T_{\mathrm{c}}$ seems to be caused by the change in the electronic density of states. The main objective of this study is to use cluster models to understand the electronic problem of $\mathrm{MgB}_{2}$. These models have been extensively used to understand the behavior of bulk materials because of the great theoretical detail that can be obtained $[6,7]$. The lowest energy equilibrium structure was obtained and corroborated with a harmonic vibrational analysis. Natural orbital analysis was performed in order to characterize the electron density of the system. Boron clusters were also considered for comparison purposes with the results obtained being in agreement with recent studies [8].

\section{METHODS}

The theoretical methods used in this study were based on ab-initio methods within the density functional theory approximation. Specific details of the procedure followed can be found elsewhere [9]. Specifically, the three parameter hybrid functional B3PW91 was used with the Dunning basis set cc-pvtz augmented with polarization functions. Hence, the method was termed B3PW91/aug-cc-pvtz. For all systems, a full geometry optimization was performed and various 


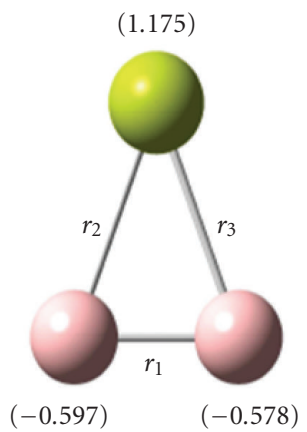

(a)

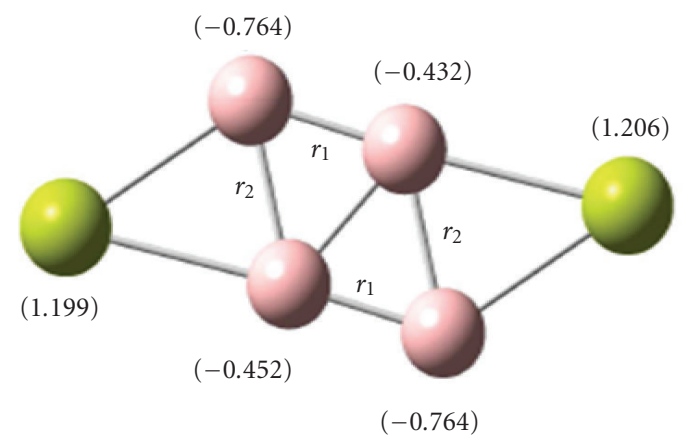

(c)

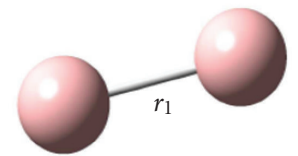

(b)

bond orbital analysis is included.

TABLe 1: Optimized bond length in angstroms.

\begin{tabular}{lccccc}
\hline $\mathrm{MgB}_{2}$ & $\left(\mathrm{MgB}_{2}\right)_{2}$ & $\left(\mathrm{MgB}_{2}\right)_{3}$ & $\mathrm{~B}_{2}$ & $\mathrm{~B}_{4}$ & $\mathrm{~B}_{6}$ \\
\hline$r_{1}=1.551$ & $r_{1}=1.726$ & $r_{1}=1.545$ & $r_{1}=1.641$ & $r_{1}=1.522$ & $r_{1}=1.797$ \\
$r_{2}=2.197$ & $r_{2}=1.581$ & $r_{2}=1.585$ & - & - & $r_{2}=1.586$ \\
- & $r_{3}=2.157$ & $r_{3}=1.730$ & - & - & $r_{3}=1.573$ \\
- & $r_{4}=2.254$ & $r_{4}=1.700$ & - & - & $r_{4}=1.541$ \\
- & - & $r_{5}=2.247$ & - & - & - \\
- & - & $r_{6}=2.323$ & - & - & - \\
- & - & $r_{7}=3.352$ & - & - & - \\
- & - & $r_{8}=2.800$ & - & - & - \\
\hline
\end{tabular}

initial geometries were used to guarantee the determination of the lowest energy equilibrium structure. Harmonic vibrational analysis was performed for each system not only to obtain the vibrational frequencies, but also to characterize the nature of the structure obtained in the potential energy surface (PES). All calculations were performed using the Gaussian 03 software package [10] installed on an SGI 200 workstation. In order to analyze the charge distribution in the system, natural bond orbital (NBO) analysis was performed on the optimized wave function using the NBO program of the Gaussian 03 package. This analysis provides an improvement to the Mulliken population analysis usually used in the description of the charge distribution.

\section{RESULTS AND DISCUSSION}

The equilibrium bond lengths of all the optimized structures are presented in Table 1 and the structures are shown in Figures 1 and 2. Figure 1(a) shows the lowest energy structure of $\mathrm{MgB}_{2}$. As in previous studies [11, 12] a nonlinear structure was obtained as the lowest energy equilibrium structure with the boron atoms at an equilibrium distance $0.09 \AA$ shorter than in the $B_{2}$ molecule (see Figure $1(b)$ ). From the NBO analysis shown in Figure 1(b), it can be observed that the electronic density is concentrated at the boron atoms, that is, the magnesium is donating electron density to the diborine. Table 2 shows the harmonic vibrational spectrum of $\mathrm{MgB}_{2}$ and $\mathrm{B}_{2}$ and it can be observed that all frequencies obtained have a positive sign which means that the global minimum was obtained. In the case of $\mathrm{MgB}_{2}$, the frequency that corresponds to the diboride vibration, frequency 1 , is $16 \%$ higher than in the $\mathrm{B}_{2}$ molecule because the bond shortening. Figures 1 (c) and 1(d) show the lowest energy for equilibrium structure of $\left(\mathrm{MgB}_{2}\right)_{2}$ and $\mathrm{B}_{4}$, respectively. Both structures show a planar arrangement of atoms, with the boron atoms in a two-dimensional unsymmetrical rhombic arrangement for $\left(\mathrm{MgB}_{2}\right)_{2}$ and a perfect square for $\mathrm{B}_{4}$. Specifically, two of the boron-boron bonds in the molecular species do not change and the other two were stretched by $9 \%$. The magnesium atoms were accommodated at a relatively long distance when compared to the separation of the boron atoms. The vibrational frequency analysis shown in Table 2 demonstrates that 


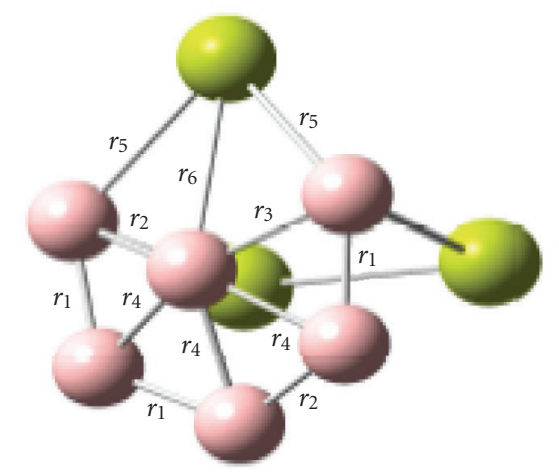

(a)

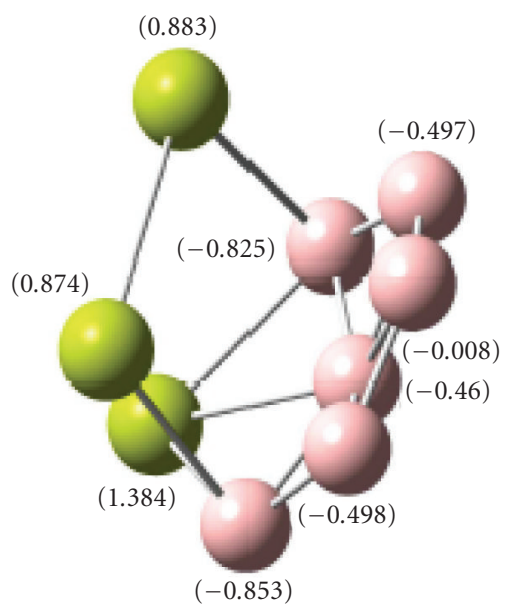

(c)

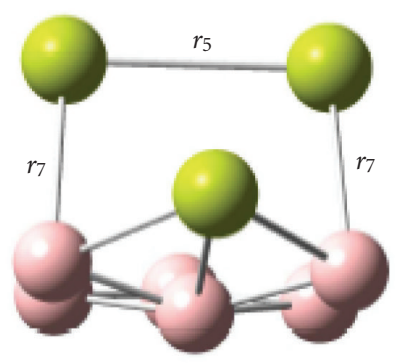

(b)

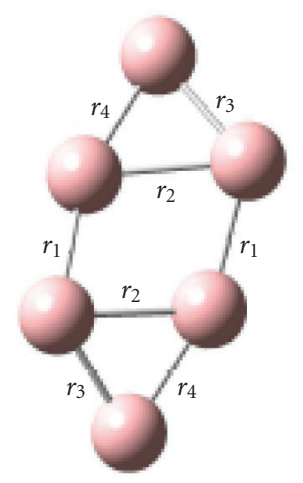

(d)

FIgURe 2: Lowest energy equilibrium structure of $\left(\mathrm{MgB}_{2}\right)_{3}$ and $\mathrm{B}_{6}$. Panel 2(a) and 2(b) show two different perspectives of $\left(\mathrm{MgB}_{2}\right)_{3}$; panel 2(c) shows the $\mathrm{NBO}$ for the lowest energy equilibrium structure of $\left(\mathrm{MgB}_{2}\right)_{3}$; and panel 2(d) shows the lowest energy equilibrium structure for $\mathrm{B}_{6}$.

the structure is a minimum in the PES. A direct comparison between the modes in the molecular species and the pure boron clusters was not possible. Figure 1(c) shows the NBO density analysis for the dimer and it can be observed that all the boron atoms forming the 2D rhombic arrangement have a negative charge. The boron atoms, which are closer to the $\mathrm{Mg}$ atoms, showed larger value of electron density than the other atoms which has the magnesium at a larger distance. Hence, the electron density is being concentrated on the boron atoms in a nonsymmetrical fashion and is being donated by the $\mathrm{Mg}$ atoms. Figure 2 shows the lowest energy equilibrium structure of $\left(\mathrm{MgB}_{2}\right)_{3}$ and $\mathrm{B}_{6}$. Boron atoms form a nonsymmetrical nonplanar arrangement. Although the $\mathrm{B}_{6}$ do not show a planar structure, the arrangement of atoms is different to the one obtained in the molecular species. The vibrational analysis shows all positive frequencies and similar to the previous system, it is not possible to correlate the frequencies between the $\mathrm{B}_{6}$ and the molecular system. The NBO analysis for the molecular systems shows a similar elec- tronic distribution to the one observed for the dimer, namely, the electronic distribution is located on the boron atoms. Two of the boron atoms show a larger electronic distribution $(-0.853)$ because of their proximity to the magnesium atoms.

\section{CONCLUSIONS}

In all cases considered, the lowest energy equilibrium structure for $\mathrm{MgB}_{2}$ clusters showed an arrangement of boron atoms that are two dimensional or quasi-two dimensional. The electron densityis concentrated on the boron atoms and hence the magnesium atoms are acting as electronic donating species. These results are in accordance to what is observed for $\mathrm{MgB}_{2}$ in the bulk [13]. If the superconductivity behavior of this material is increased by the larger electronic density in a specific plane, then it can be expected that this clusters should exhibit a similar superconducting behavior. 
TABle 2: Harmonic vibrational frequencies in $\mathrm{cm}^{-1}$. Number in parenthesis is used to label the frequency.

\begin{tabular}{|c|c|c|c|c|c|}
\hline $\mathrm{MgB}_{2}$ & $\left(\mathrm{MgB}_{2}\right)_{2}$ & $\left(\mathrm{MgB}_{2}\right)_{3}$ & $\mathrm{~B}_{2}$ & $\mathrm{~B}_{4}$ & $\mathrm{~B}_{6}$ \\
\hline (1) 452 & (1) 64 & (1) 70 & (1) 962 & (1) 313 & (1) 184 \\
\hline (2) 496 & (2) 191 & (2) 138 & - & (2) 352 & (2) 305 \\
\hline (3) 1132 & (3) 248 & (3) 146 & - & (3) 1063 & (3) 346 \\
\hline - & (4) 253 & (4) 175 & - & (4) 1182 & (4) 381 \\
\hline- & (5) 324 & (5) 254 & - & (5) 1276 & (5) 560 \\
\hline - & (6) 428 & (6) 293 & - & (6) 1277 & (6) 563 \\
\hline - & (7) 448 & (7) 306 & - & - & (7) 713 \\
\hline - & (8) 578 & (8) 336 & - & - & (8) 838 \\
\hline - & (9) 606 & (9) 380 & - & - & (9) 1074 \\
\hline - & (10) 889 & (10) 431 & - & - & (10) 1120 \\
\hline - & 1080 & (11) 460 & - & - & 1261 \\
\hline - & (12) 1104 & (12) 489 & - & - & 1320 \\
\hline - & - & (13) 573 & - & - & - \\
\hline - & - & (14) 581 & - & - & - \\
\hline - & - & (15) 696 & - & - & - \\
\hline - & - & (16) 725 & - & - & - \\
\hline - & - & (17) 819 & - & - & - \\
\hline - & - & ) 973 (18) & - & - & - \\
\hline - & - & 1078 (19) & - & - & - \\
\hline - & - & 1193 (20) & - & - & - \\
\hline- & - & 1221 (21) & - & - & - \\
\hline
\end{tabular}

\section{ACKNOWLEDGMENT}

This work is supported by grants from the NIH-SCORE (Grant No. GM008103-03) and NIH-COBRE (Grant No. P2RR16439-01) programs.

\section{REFERENCES}

[1] S. L. Bud'ko, G. Lapertot, C. Petrovic, C. E. Cunningham, N. Anderson, and P. C. Canfield, "Boron isotope effect in superconducting $\mathrm{MgB}_{2}$," Physical Review Letters, vol. 86, no. 9, pp. 1877-1880, 2001.

[2] J. Kortus, "Current progress in the theoretical understanding of $\mathrm{MgB}_{2}$," Physica C, vol. 456, no. 1-2, pp. 54-62, 2007.

[3] H. J. Choi, D. Roundy, H. Sun, M. L. Cohen, and S. G. Louie, "The origin of the anomalous superconducting properties of $\mathrm{MgB}_{2}$," Nature, vol. 418, no. 6899, pp. 758-760, 2002.

[4] H. Y. Zhai, H. M. Christen, L. Zhang, et al., "Superconducting magnesium diboride films on $\mathrm{Si}$ with $T_{c 0} \sim 24 \mathrm{~K}$ grown via vacuum annealing from stoichiometric precursors," Applied Physics Letters, vol. 79, no. 16, pp. 2603-2605, 2001.

[5] G. L. Zhao, A. Pullen, and D. Bagayoko, "The metallic nature of boron layers in magnesium diboride," International Journal of Modern Physics B, vol. 17, no. 31-32, pp. 5905-5910, 2003.

[6] J. Zhao and R.-H. Xie, "Cluster-assembled materials based on $\mathrm{Na}_{6} \mathrm{~Pb}$," Physical Review B, vol. 68, no. 3, Article ID 035401, 5 pages, 2003.

[7] X. Gu, S. Bulusu, X. Li, et al., " $\mathrm{Au}_{34}{ }^{-}$: a fluxional core-shell cluster," Journal of Physical Chemistry C, vol. 111, no. 23, pp. 8228-8232, 2007.
[8] A. N. Alexandrova, A. I. Boldyrev, H.-J. Zhai, and L.-S. Wang, "All-boron aromatic clusters as potential new inorganic ligands and building blocks in chemistry," Coordination Chemistry Reviews, vol. 250, no. 21-22, pp. 2811-2866, 2006.

[9] M. C. Vicens and G. E. López, "Density functional studies of cation-water complexes," Journal of Computational Chemistry, vol. 21, no. 1, pp. 63-68, 2000.

[10] M. J. Frisch, G. W. Trucks, H. B. Schlegel, et al., Gaussian Inc Wallingford CT, 2004.

[11] S. Zhang, P. Yang, and S. Li, "Geometries and properties of adenine- $\mathrm{BH}_{3}$ complex: an investigation with density functional theory (DFT) method," Journal of Molecular Structure: THEOCHEM, vol. 677, no. 1-3, pp. 161-166, 2004.

[12] E. P. F. Lee and T. G. Wright, "The ground state and heat of formation of $\mathrm{MgB}_{2}$," Journal of Physical Chemistry A, vol. 108, no. 36, pp. 7424-7428, 2004.

[13] Ş. Erkoç, "Structural and electronic properties of magnesium diboride," International Journal of Modern Physics C, vol. 14, no. 4, pp. 483-489, 2003. 

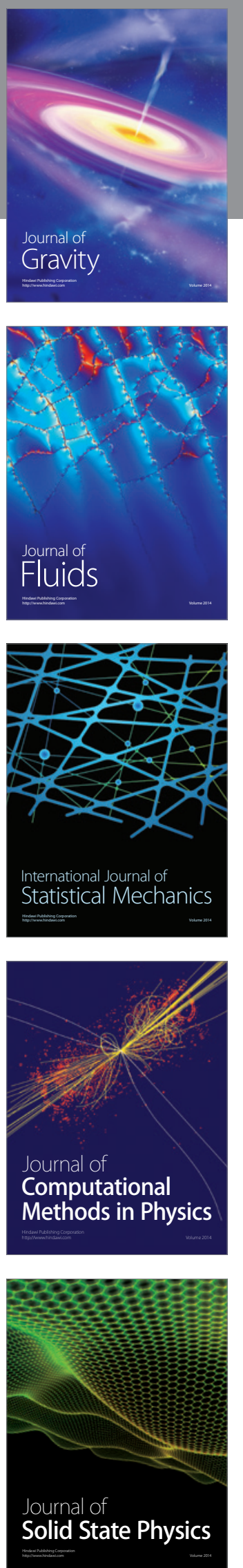

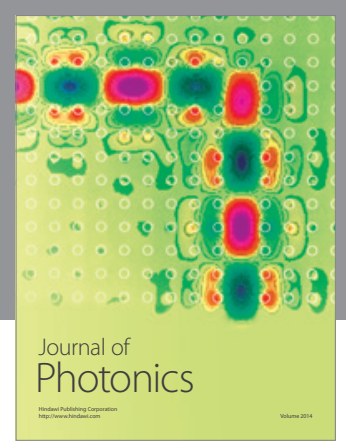

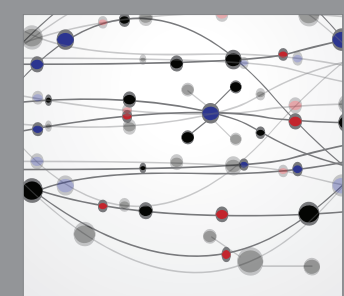

The Scientific World Journal
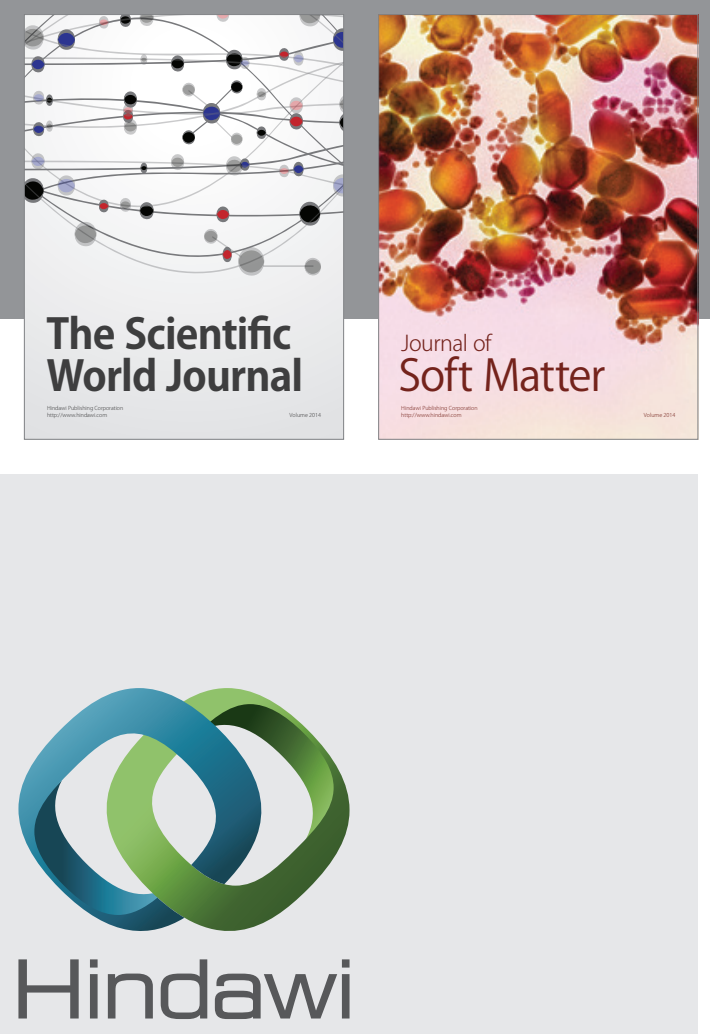

Submit your manuscripts at

http://www.hindawi.com
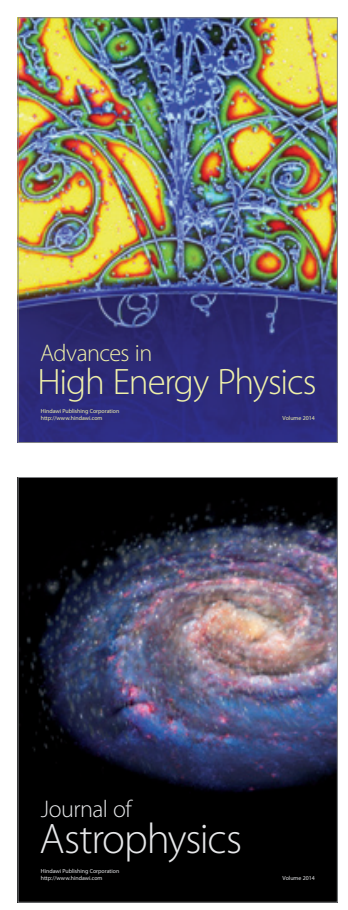
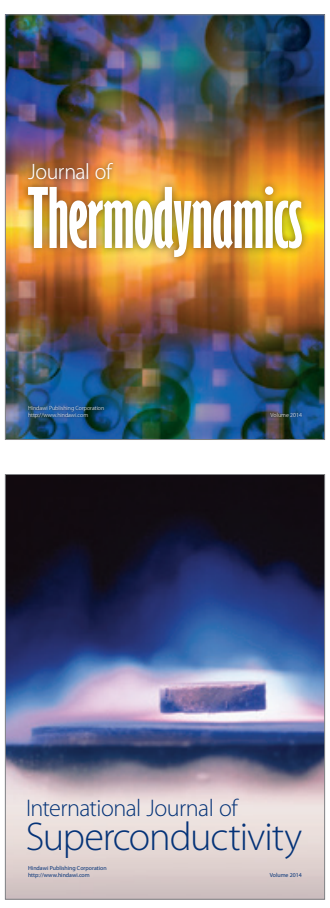
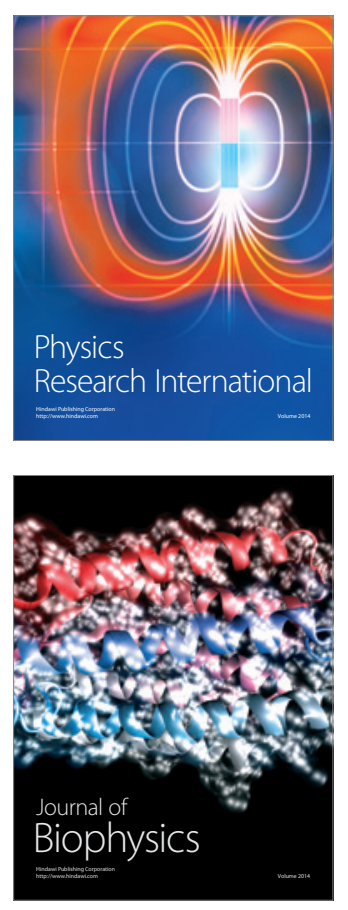
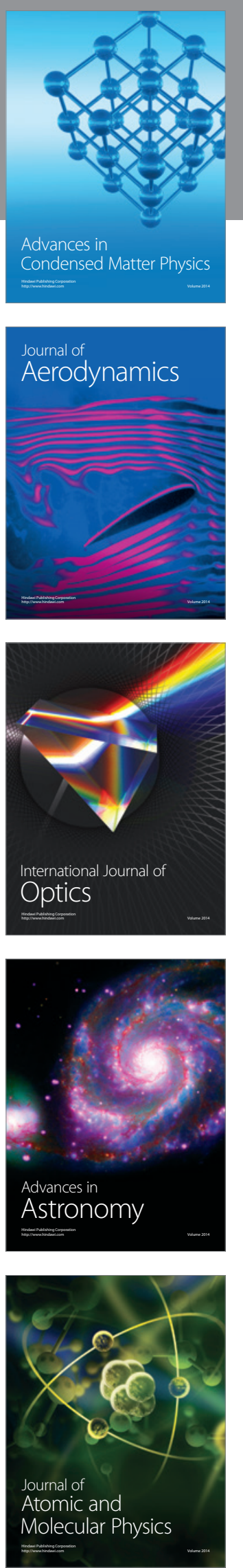